\title{
Disposable Nitrate-Selective Optical Sensor Based on Fluorescent Dye
}

\author{
Giyoung Kim, Kenneth A. Sudduth ${ }^{2}$, Sheila A. Grant ${ }^{3}$, Newell R. Kitchen ${ }^{2}$ \\ ${ }^{1}$ National Academy of Agricultural Science, 249 Seodun-dong, Suwon, Korea, ${ }^{2}$ USDA-ARS Cropping Systems and Water \\ Quality Research Unit, Columbia, MO, USA, ${ }^{3}$ Biological Engineering,University of Missouri, Columbia, MO, USA
}

Received: June $23^{\text {th }}, 2012$; Revised: June $29^{\text {th }}, 2012$; Accepted: June $29^{\text {th }}, 2012$

\section{Abstract}

Purpose: This study was performed to develop a simple, disposable thin-film optical nitrate sensor. Methods: The sensor was fabricated by applying a nitrate-selective polymer membrane on the surface of a thin polyester film. The membrane was composed of polyvinylchloride (PVC), plasticizer, fluorescent dye, and nitrate-selective ionophore. Fluorescence intensity of the sensor increased on contact with a nitrate solution. The fluorescence response of the optical nitrate sensor was measured with a commercial fluorospectrometer. Results: The optical sensor exhibited linear response over four concentration decades. Conclusions: Nitrate ion concentrations in plant nutrient solutions can be determined by direct optical measurements without any conditioning before measurements.

Keywords: Optical sensor, Nitrate-selective, Ionophore, Fluorescent Dye

\section{Introduction}

Hydroponics has several advantages over conventional soil-based plant culture. Besides the increase in yields and quality of crops, the use of hydroponic technology reduces chemical usage and toxic residues by eliminating soil fumigation and weeding. Hydroponics also conserves water and reduces environmental pollution. Using a closed hydroponic system which recycles the nutrient solution achieves further conservation of water and environmental benefits.

Despite these advantages, the expanded use of closed hydroponic systems in Korea is hindered by technical problems. The major obstacle to developing closed hydroponic systems is the difficulty in measuring the amount of individual ion components in the hydroponic nutrient solution. Because crop nutrient needs vary with growth stage and environment, controlling hydroponic nutrients simply based on the level of electrical conductivity (EC)

\footnotetext{
*Comesponding author: Kenneth Sudduth

Tel: +1-573-882-1114 x303; Fax: +1-573-882-1115

E-mail: sudduth@missouri.edu
}

and $\mathrm{pH}$ could cause unbalanced nutrition and lead to nutrient deficiencies or salt accumulation. To fulfill the specific needs of the crops during different growth stages, precise formulation of the nutrient solution is essential. Measuring the concentration of individual ions in the nutrient solution is necessary to monitor and control the formulation of the nutrient solution.

One of the most widely used types of ion sensors is the ion selective electrode (ISE). ISEs are based on ionophores, chemical compounds that selectively bind to ions. ISEs have been widely used for in-situ analysis because they are highly selective and easy to use. Also, ISEs have a low initial setup cost and are less expensive than atomic adsorption spectrophotometry (AAS) or Ion Chromatography (IC). However, conventional ISEs require an internal reference electrode and liquid filling solution. The internal filling solution hampers maintenance and miniaturization of the device. For these reasons much research has been conducted to develop ISEs that have a solid interface between the sensing membrane and the solution. Some of the approaches to developing planar solid state electrodes (SSEs) are the screen-printing tech- 
nology based thick-film sensor (Koncki et al., 1999), photocured technique based thin-film sensor (Heng and Hall, 2001), micromachining technology based miniaturized ISE sensor chip (Uhlig et al., 1996; Zhu et al., 2000), miniaturized electrode coupled with a portable measuring instrument (Ro $\beta$ et al., 1995), and array-type multi-ion sensors (Lee et al., 2000; Mourzina et al., 2001). However, wide acceptance of these solid-state ISEs has been hindered by their inherent noise and drift problems.

Another common type of ionophore-based sensor for in-situ analysis is the optical sensor. Recent developments in understanding the theoretical working principles of these sensors provide possibilities to devise rapid and low cost in-situ ion analysis methods. Some optical sensors utilize a potential sensitive dye (PSD) that changes its optical properties in response to microenvironment changes (Mohr et al., 1997). Research has been conducted to develop optical sensors for various ions, such as nitrate (Mohr et al., 1995), nitrite (Mohr and Wolfbeis, 1996), chloride (Huber et al., 1999), ammonium (Stromberg and Hulth, 2005), and calcium (Ryan and Urayama, 2012). Most of these optical sensors used tridodecylmethyl ammonium chloride (TDMACl) as an ion carrier. This compound is highly selective to the chloride ion that is commonly found in tap water. To overcome interference from chloride ions, some researchers developed optical sensors based on ion-selective PSD alone without using the anion carrier (Huber et al., 2001).

The goal of this study was to develop a nitrate-selective optical sensor based on PSD with a nitrate-selective ionophore. A disposable type thin-film optical nitrate sensor was fabricated and the performance was assessed by analysing analytical characteristics of the sensor.

\section{Materials and Methods}

The fluorescent dyes, fluorescein octadecylester (FOE) and 4-(4-(dihexadecylamino)styryo-N-methylpyridinium iodide (DiA) were acquired from Invitrogen (Carlsbad, CA, USA). The nitrate ionophores, tridodecylmethylammonium nitrate (TDMANO3) and tetraoctylammonium nitrate (TOAN) were obtained from Fluka (Buchs, Switzerland). The plasticizer bis (2-ethylhexyl) sebacate (DOS) and the membrane substrate copolymer of vinyl chloride, vinyl acetate, and vinyl alcohol (PVC-CO) were from Fluka (Buchs, Switzerland). The solvent tetrahydrofuran (THF) was also from Fluka (Buchs, Switzerland). The thin film support, Mylar type $175 \mu \mathrm{m}$ thick polyester foil was acquired from Dupont (Wilmington, DE, USA). Sodium nitrate, sodium chloride, and disodium sulphate used to prepare test solutions were of analytical grade. Deionized water was used to prepare buffers.

Membrane solutions were manually prepared by mixing ionophore, PSD, plasticizer, PVC copolymer, and THF. Five different sensor compositions were tested. Sensors based on FOE (S1, S2) were composed of $1.05 \mathrm{mg}$ of TDMANO3 or TOAN, $1.15 \mathrm{mg}$ of FOE, $32.55 \mathrm{mg}$ of DOS, and $15.25 \mathrm{mg}$ of PVC copolymer in $4.5 \mathrm{ml}$ of THF. Sensor membranes (S3-S5) made with DiA was composed of $1.05 \mathrm{mg}$ of TOAN, $32.55 \mathrm{mg}$ of DOS, $15.25 \mathrm{mg}$ of PVC copolymer, and different ratios of DiA in $4.5 \mathrm{ml}$ of THF. Table 1 shows the composition of the five nitrate sensor membranes. In order to make the nitrate-selective optical sensor, $0.2 \mathrm{ml}$ of the membrane cocktail solution was dropped onto the polyester foil. The polyester film was placed into a closed container that provided a THF saturated environment for $30 \mathrm{~min}$. After the membrane solution was evenly distributed on the surface film, the film was placed in the air for $24 \mathrm{hrs}$ to dry and form a solid-state membrane.

Responses of the optical sensors to different concentrations of nitrate were measured with a spectrofluorometer Jasco FP-750 (Jasco, Inc., Easton, MD, USA), which has a wavelength range of $220 \mathrm{~nm}$ to $730 \mathrm{~nm}$ provided by a xenon lamp. Data from the spectrofluoro-

Table 1. Compositions of the five nitrate optical sensor membranes using different fluorescent dyes and ionophores

\begin{tabular}{|c|c|c|c|c|}
\hline Sensor & $\mathrm{PSDa}(\mathrm{mg})$ & lonophore & Plasticizer & Polymer \\
\hline S1 & $\mathrm{FOE}^{\mathrm{b}}$ & TDMANO3 $^{\mathrm{C}}$ & $\operatorname{DOS}^{d}$ & PVC-CO ${ }^{e}$ \\
\hline S2 & FOE & TOAN $^{f}$ & DOS & PVC-CO \\
\hline S3 & $\mathrm{DiA}^{\mathrm{g}}(0.4)$ & - & DOS & PVC-CO \\
\hline S4 & DiA (0.4) & TOAN & DOS & PVC-CO \\
\hline S5 & DiA (0.8) & TOAN & DOS & PVC-CO \\
\hline
\end{tabular}

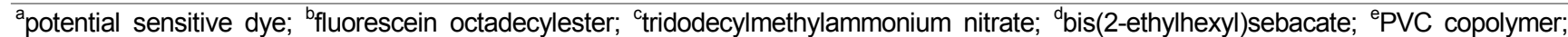
tetraoctylammonium nitrate; ${ }^{\mathrm{f}}$ 4-(4-(dihexadecylamino)styryo-N-methylpyridinium iodide. 
meter were recorded on a computer connected via a RS-232 interface. Excitation wavelengths for FOE and DiA were $504 \mathrm{~nm}$ and $460 \mathrm{~nm}$, respectively. Fluorescence emission signals were measured with 'low' sensitivity settings of the spectrofluorometer for both PSD based membranes to reduce noise. To measure the fluorescence response, the thin-film optical sensor was placed into a $4.5-\mathrm{ml}, 1-\mathrm{cm}$ path-length cuvette at a $45^{\circ}$ angle and $3 \mathrm{ml}$ of sample solution was added (Fig. 1).

The performance of the optical sensors was evaluated by the slope, response time, repeatability, and selectivity coefficients. For fluorescent characteristic tests, standard solutions of $\mathrm{NaNO}_{3}$ were prepared by weighing the corresponding analytical grade salt. All solutions were prepared by using double-distilled water. Fluorescence measurements with the optical sensor were made using sample solutions of in the concentration range $10^{-5}$ to $10^{-1}$ mol/l. Selectivity coefficients were determined by the separate solution method. To find selectivity coefficients, the calibration curve of the optical sensor to the nitrate ion was acquired first. Then responses to each interfering ion were measured.

For repeatability tests, the sensor as washed with distilled water before measurements to obtain a reproducible constant fluorescence. For performance tests three measurements were made and all measurements were carried out at room temperature.

\section{Results and Discussion}

The fluorescence response of the optical nitrate sensor $\mathrm{S} 5$ is shown in Fig. 2. The emission maximum of DiA in the

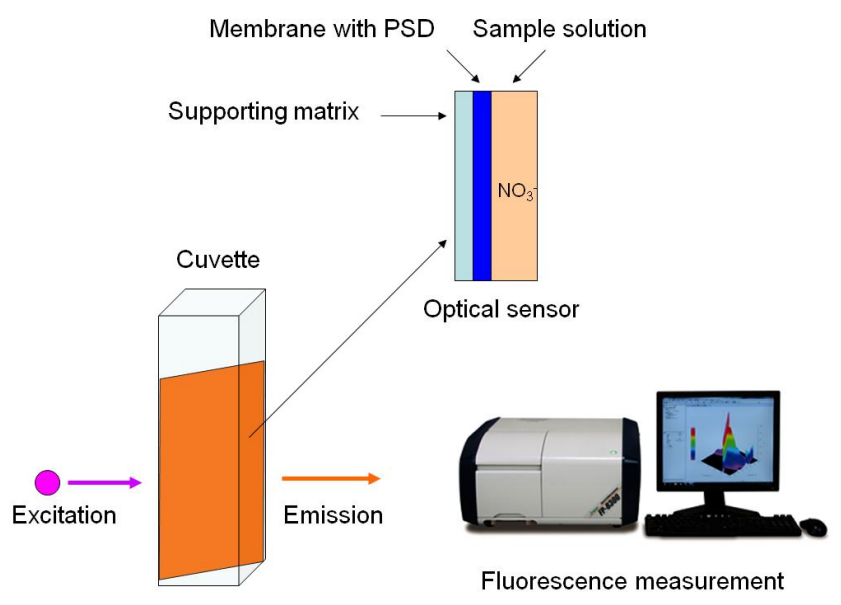

Figure 1. Schematic diagram of the disposable optical sensor. sensor membrane was between $552-560 \mathrm{~nm}$. The wavelength at the emission maximum gradually shifted from $552 \mathrm{~nm}$ for $10^{-5} \mathrm{~mol}$ to $560 \mathrm{~nm}$ for $10^{-1} \mathrm{~mol}$ of nitrate. The fluorescence intensity increased as the concentration of nitrate ions in the solution increased. Similar fluorescence intensity increase with respect to an increased concentration of nitrate ions was observed for the other sensors containing DiA (S3, S4). The emission maximum of FOEbased nitrate sensors S1 and S2 was $545 \mathrm{~nm}$. The fluorescence intensity of sensor S1 and S2 also increased as the concentration of nitrate ions in the solution increased. However, the response of each of the FOE-based nitrate sensors (S1, S2) was irreversible when the sensor was placed into plain distilled water. The fluorescence intensity stayed high after being exposed to a high nitrate concentration samples and did not return to a background level. Because of this reproducibility issue, further performance tests were conducted only for sensors containing DiA.

The reversibility of fluorescence response changes over time for sensor S5 are shown in Fig. 3. The fluorescence intensity increased upon contact with nitrate solution and then decreased upon exposure to distilled water. The response time of sensor S5 was less than $3 \mathrm{~min}$, a value similar to other research results (Mohr et al., 1997; Huber et al., 2001).

Fig. 4 shows the calibration results of the optical nitrate sensors (S3-S5). The detection limit of sensor S4 was $10^{-3}$ mol. Detection limits of sensors S3 and S5 were lower than $10^{-4} \mathrm{~mol}$, which sufficiently covers the range of nitrate ion concentrations in typical hydroponic nutrient solutions $\left(10^{-2} \mathrm{~mol}\right)$. The higher content of dye (DiA) in sensor S5 gave a larger increase over the background

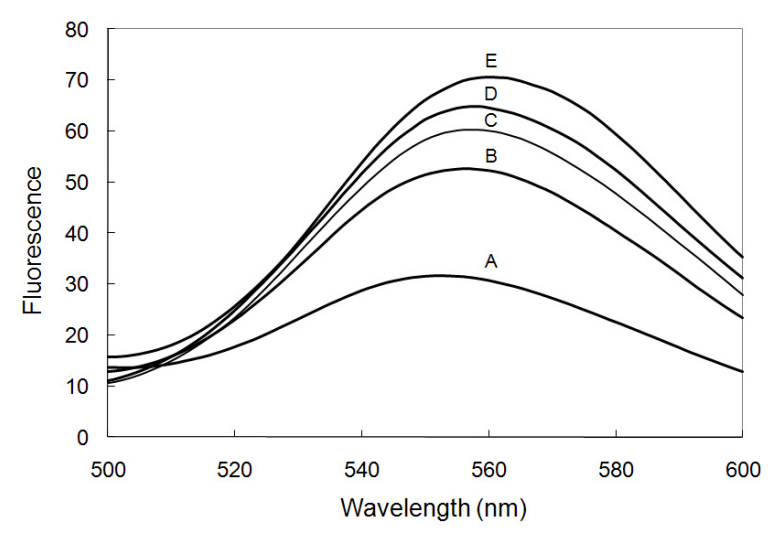

Figure 2. Fluorescence emission spectra of the optical nitrate sensor S5 when exposed to: A, $10^{-5}$ mol nitrate; $B, 10^{-4}$ mol nitrate; C, $10^{-3} \mathrm{~mol}$ nitrate; $\mathrm{D}, 10^{-2} \mathrm{~mol}$ nitrate; and $\mathrm{E}, 10^{-1} \mathrm{~mol}$ nitrate. 


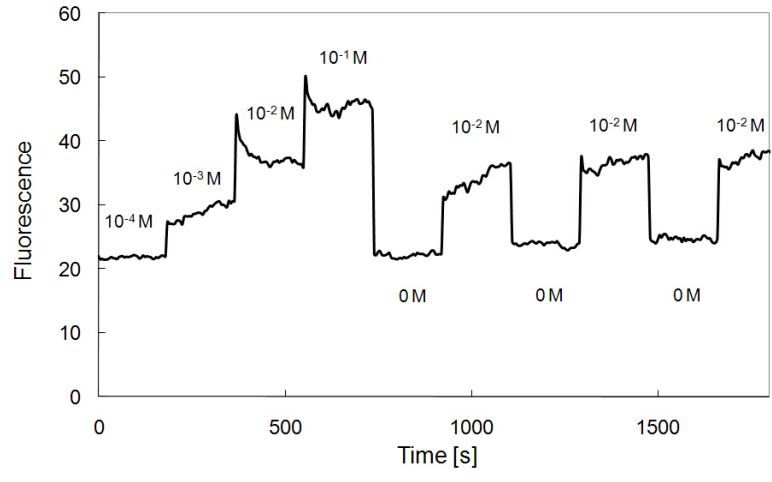

Figure 3. Fluorescence changes over time and concentration of nitrate for sensor S5.

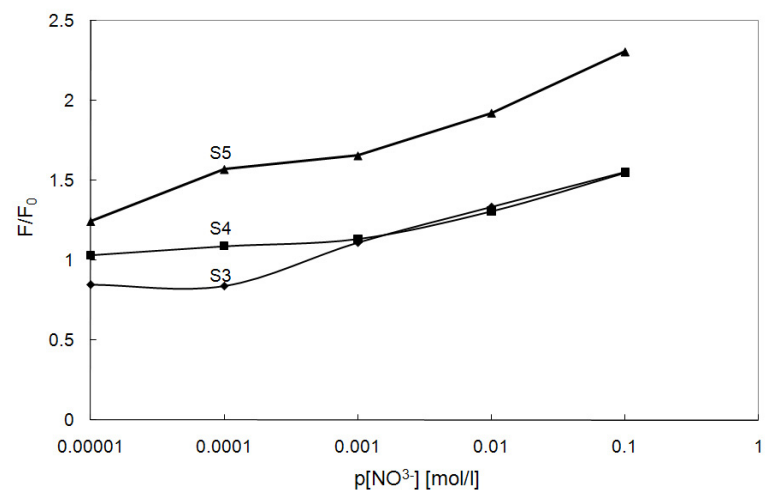

Figure 4. Calibration curves of optical sensors S3, S4, and S5. The fluorescent dye, DiA, contents were $0.4 \mathrm{mg}(\mathrm{S} 3, \mathrm{~S} 4)$ and 0.8 $\mathrm{mg}$ (S5). Sensor S3 did not contain the nitrate ionophore TOAN. (F/FO : relative fluorescence intensity relative to baseline intensity).

signal ( 0 mol of nitrate). At $10^{-2} \mathrm{~mol}$ of nitrate, the increases in the relative fluorescence intensity of S3 and S4 were $33 \%$ and $30 \%$, respectively. The increase in the relative fluorescence intensity of S5 from $0 \mathrm{~mol}$ to $10^{-2}$ mol of nitrate was $92 \%$.

Calibrations of the nitrate optical sensor were conducted in the presence of typical interfering ions to investigate the selectivity of sensor S5. Fig. 5 shows fluorescence response of sensor 55 to typical interfering ions for nitrate ion sensors. The optical nitrate optical sensor did not seem to exhibit interference from chloride and sulphate, two most strongly interfering ions for ISE-type nitrate sensors.

\section{Conclusions}

In this study, we developed a simple, disposable thin-

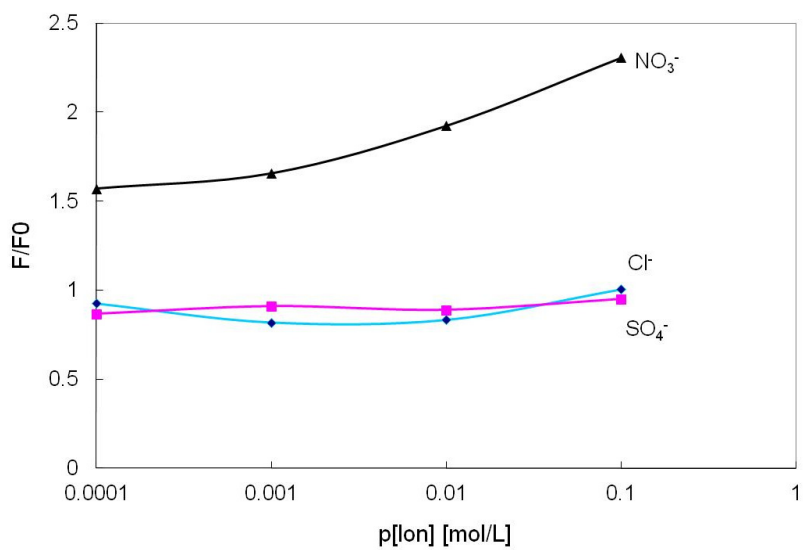

Figure 5. Calibration curves of optical sensor S5 to nitrate and to interfering chloride and sulfate ions. (F/FO: relative fluorescence intensity relative to baseline intensity).

film optical nitrate sensor. The sensor fabricated by using a nitrate-selective polymer membrane showed proportional increase of fluorescence intensity on contact with different concentrations of nitrate solution. Although further improvement is necessary, the DiA optical nitrate sensor showed acceptable performance. Successful development of an optical nitrate sensor based on this approach could provide an affordable and valuable tool for developing and promoting closed hydroponic systems. The direct fluorescence measurement capability of such an optical sensor could be used to control nutrient solution nitrate levels in an on-line mode.

Also, ionophore-based optical sensors are highly selective, rapid and need few matrix modifications to conduct analyses. These characteristics make ionophore-based optical sensors ideal for clinical and biological use and for in-situ analysis of environmental samples such as soil and water. The disposable optical nitrate sensor developed in this study could easily be adapted to mass production of simple, small, and cheap sensors. On-line ion analysis systems based on this optical ion-sensor technology are promising for in-situ determination of ion concentrations in hydroponic solutions.

\section{Conflict of Interest}

The authors have no conflicting financial or other interests. 


\section{Acknowledgement}

This study was carried out with the support of "Research Program for Agricultural Science \& Technology Development (Project No. PJ006812)", National Academy of Agricultural Science, Rural Development Administration, Republic of Korea.

\section{References}

Heng, L. Y. and E. A. H. Hall. 2001. Assessing a photocured self-plasticised acrylic membrane recipe for $\mathrm{Na}^{+}$and $\mathrm{K}^{+}$ion selective electrodes. Analytica Chimica Acta 443:25-40.

Huber, C., T. Werner, C. Krause and O. S. Wolfbeis. 1999. Novel chloride-selective optode based on polymerstabilised emulsions doped with a lipophilic fluorescent polarity-sensitive dye. Analyst 124:1617-1622.

Huber, C., I. Klimant, C. Krause, T. Werner and O. S. Wolfbeis. 2001. Nitrate-selective optical sensor applying a lipophilic fluorescent potential-sensitive dye. Analytica Chimica Acta 449:81-93.

Koncki, R., S. Glab, J. Dziwulska, I. Palchetti and M. Mascini. 1999. Disposable strip potentiometric electrodes with solvent-polymeric ion-selective membranes fabricated using screen-printing technology. Analytica Chimica Acta 385:451-459.

Lee, D. K., H. J. Lee, G. S. Cha, H. Nam and K. J. Paeng. 2000. Ion chromatography detector based on solid-state ion-selective electrode array. Journal of Chromatography A 902:337-343.

Mohr, G. J., T. Werner and O. S. Wolfbeis. 1995. Application of a novel lipophilized fluorescent dye in an optical nitrate sensor. Journal of Fluorescence 5(2): 135-138.

Mohr, G. J. and O. S. Wolfbeis. 1996. Optical nitrite sensor based on a potential-sensitive dye and a mitrite selective carrier. Analyst 121:1489-1494.

Mohr, G. J., F. Lehmann, R. Östereich, I. Murkovic and O. S. Wolfbeis. 1997. Investigation of potential-sensitive fluorescent dyes for application in nitrate sensitive polymer membranes. Fresenius' Journal of Analytical Chemistry 357:284-291.

Mourzina, Y. G., J. Schubert, W. Zander, A. Legin, Y. G. Vlasov, H. Luth and M. J. Schoning. 2001. Development of multisensor systems based on chalcogenide thin film chemical sensors for the simultaneous multicomponent analysis of metal ions in complex solutions. Electrochimca Acta 47:251-258.

Ro $\beta$, B., G. Steiner, M. Kiesshauer, M. Bradter and K. Cammann. 1995. Instrument with integrated sensors for a rapod determination of inorganic ions. Sensors and Actuators B: Chemical 26:380-383.

Ryan, J and P. Urayama. 2012. Characterizing the dualwavelength dye indo- 1 for calcium-ion sensing under pressure. Analytical Methods 4:80-84.

Stromberg, N and S. Hulth. 2005. Assessing an imaging ammonium sensor using time correlated pixel-bypixel calibration. Analytica Chimica Acta 550:61-68.

Uhlig, A., F. Dietrich, E. Lindner, U. Schnakenberg and R. Hintsche. 1996. Miniaturised ion-selective sensor chip for potassium measurement in a biomedical application. Sensors and Actuators B: Chemical 34:252-257.

Zhu, J. Z., J. F. Xie, D. R. Lu, G. X. Zhang and Z. R. Zhang. 2000. Micromachined glucose sensor and $\mathrm{K}^{+}$ISE based on containment array. Sensors and Actuators B: Chemical 65:157-159. 